PROCEEDINGS OF THE

AMERICAN MATHEMATICAL SOCIETY

Volume 131, Number 11, Pages 3309-3317

S 0002-9939(03)06945-4

Article electronically published on February 14, 2003

\title{
SPECIAL VALUES OF ELLIPTIC FUNCTIONS AT POINTS OF THE DIVISORS OF JACOBI FORMS
}

\author{
YOUNGJU CHOIE AND WINFRIED KOHNEN
}

(Communicated by David E. Rohrlich)

\begin{abstract}
The main result of the paper gives an explicit formula for the sum of the values of even order derivatives with respect to $z$ of the Weierstrass $\wp$-function $\wp(\tau, z)$ for the lattice $\mathbf{Z} \tau \oplus \mathbf{Z}$ (where $\tau$ is in the upper half-plane) extended over the points in the divisor of $\phi(\tau, \cdot)$ (where $\phi(\tau, z)$ is a meromorphic Jacobi form) in terms of the coefficients of the Laurent expansion of $\phi(\tau, z)$ around $z=0$.
\end{abstract}

\section{INTRODUCTION}

Classically, the theory of complex multiplication asserts that the value of the usual elliptic modular function $j$ at an imaginary quadratic number $\tau$ is an algebraic integer and generates a ring class field of $\mathbf{Q}\left(\sqrt{d_{\tau}}\right)$ where $d_{\tau}<0$ is the discriminant of a non-trivial integral quadratic equation satisfied by $\tau$. These special values also play an important role in modern number theory (cf. e.g. the papers [6] 7 ] by Gross-Zagier, 2] by Borcherds, [10] by Zagier and others).

A question not completely unreasonable then seems to be if there are other natural points $\tau$ in the upper half-plane $\mathcal{H}$ such that $j(\tau)$ is algebraic and has interesting arithmetic properties. Of course, by a well-known theorem of Schneider such points cannot be algebraic, and so at first sight a search appears to be rather hopeless. On the other hand, a little thought reveals that if $f$ is a non-zero elliptic modular function of weight $k$ with Fourier coefficients in a field $K \subset \mathbf{C}$, then $j(\tau)$ indeed is algebraic over $K$ for any point $\tau$ in the divisor of $f$.

In [3], a weighted average sum of the values of $j_{1}:=j-744$ (and of a sequence of modular functions $j_{n}, n \geq 2$, related to $j$ ) over such points was studied. In particular, among several other things an explicit formula for these average sums in terms of the Fourier coefficients of $f$ was obtained.

On the other hand, the values of elliptic functions at division points of the associated lattice also are important in number theory. For example, for $\tau \in \mathcal{H}$ let $L_{\tau}:=\mathbf{Z} \tau \oplus \mathbf{Z}$ be the lattice generated by $\tau$ and 1 and denote by $w(\tau, \cdot)$ the associated Weber function (i.e. essentially the Weierstrass $\wp$-function $\wp(\tau, \cdot)$ for $L_{\tau}$ properly normalized to be homogeneous of degree zero with respect to $L_{\tau}$ ). Then it is a classical fact that for $\tau$ imaginary quadratic the value of $w(\tau, \cdot)$ at a non-trivial $N$-division point of $L_{\tau}$ is algebraic, and in fact together with $j(\tau)$ generates the ray

Received by the editors May 24, 2002.

2000 Mathematics Subject Classification. Primary 11F03, 11 G05.

(C)2003 American Mathematical Society 
class field of $\mathbf{Q}\left(\sqrt{d_{\tau}}\right)$ of conductor $N$ (cf. e.g. [9, chap. 10, sect. 1, Thm. 2 and sect. 2, Cor. to Thm. 7]).

In the above context, following [3] it appears to be quite reasonable to also look for points $z \in \mathbf{C}$ other than division points such that $\wp(\tau, z)$ is algebraic, and to study the corresponding values. Natural candidates here seem to be the points in the divisor of $\phi(\tau, \cdot)$, where $\phi(\tau, z)(\tau \in \mathcal{H}, z \in \mathbf{C})$ is a Jacobi form.

In the present paper we will indeed give a formula analogous to the abovementioned one of $\left[3\right.$ in the case of elliptic functions, with $j_{n}$ replaced by $\wp_{n}$ (essentially the $(2 n-2)$-th partial derivative of $\wp$ with respect to $z)$ and $f$ replaced by a Jacobi form $\phi$. More precisely, let $\phi(\tau, z)(\tau \in \mathcal{H}, z \in \mathbf{C})$ be a meromorphic Jacobi form of weight $k \in \mathbf{Z}$ and index $m \in \mathbf{Z}$ with a $K$-rational $q$-expansion $\left(q:=e^{2 \pi i \tau}\right)$. Fix $\tau \in \mathcal{H}$ and suppose that $\phi(\tau, \cdot)$ is a non-zero meromorphic function in $z$. Let $g_{2}(\tau)$ and $g_{3}(\tau)$ be the usual Weierstrass invariants attached to $L_{\tau}$. Then the values $\wp_{n}(\tau, z)$ where $z$ is in the divisor of $\phi(\tau, \cdot)$ and $z \notin L_{\tau}$ are algebraic over $K\left(g_{2}(\tau), g_{3}(\tau)\right.$ ) (section 3, Proposition 1), and we express a weighted average sum of these values explicitly in terms of the Laurent series coefficients of $\phi(\tau, \cdot)$ around $z=0$ and in terms of values of Eisenstein series at $\tau$ (section 4, Theorem).

Formally, in analogy with a corresponding result of [3, as is quite clear our Theorem can also be restated using the exponents of a product expansion of $\phi(\tau, \cdot)$ in terms of the functions $1-z^{n}(n \geq 1)$. We shall make some short comments on this in section 5 and as an example explicitly state the result in the case of $\phi_{10,1}$ (section 5, Proposition 3).

Our method of proof is similar to that in [3. In other words, we shall use a $\wp_{n^{-}}$ weighted version of the classical well-known valence formula for elliptic functions (respectively for Jacobi forms, in the latter case; cf. [5, chap. I, sect.1, Thm. 1.2]).

Notations. For $\tau \in \mathcal{H}, z \in \mathbf{C}$ we shall write $q=e^{2 \pi i \tau}, \zeta=e^{2 \pi i z}$. We sometimes write $\rho=e^{2 \pi i / 3}$. The letter $K$ denotes a subfield of $\mathbf{C}$, fixed throughout.

\section{Preliminaries on Meromorphic Jacobi Forms AND ELLIPTIC FUNCTIONS}

We shall start by recalling the definition of a meromorphic Jacobi form (cf. [1, [5]). Let $k$ and $m$ be fixed integers. Then a function $\phi$ on $\mathcal{H} \times \mathbf{C}$ is called a meromorphic Jacobi form of weight $k$ and index $m$ if

i) $\phi$ is meromorphic on $\mathcal{H} \times \mathbf{C}$,

ii) $\phi\left(\frac{a \tau+b}{c \tau+d}, \frac{z}{c \tau+d}\right)=(c \tau+d)^{k} \exp \left(2 \pi i m \frac{c z^{2}}{c \tau+d}\right) \phi(\tau, z)\left(\forall\left(\begin{array}{ll}a & b \\ c & d\end{array}\right) \in \Gamma_{1}:=S L_{2}(\mathbf{Z})\right)$ and $\phi(\tau, z+\lambda \tau+\mu)=\exp \left(-2 \pi i m\left(\lambda^{2} \tau+2 \lambda z\right)\right) \phi(\tau, z)\left(\forall(\lambda, \mu) \in \mathbf{Z}^{2}\right)$,

iii) $\phi$ has a meromorphic $q$-expansion of the form

$$
\phi(\tau, z)=\sum_{n \geq h} c_{n}(z) q^{n} \quad\left(0<|\zeta|<A, 0<|q|<B|\zeta|^{N}\right)
$$

where $A, B>0, h \in \mathbf{Z}$ and $N \in \mathbf{N}$ are constants and the coefficients $c_{n}(z)$ for all $n$ are contained in the field $\mathbf{C}(\zeta)$ of complex rational functions.

Applying ii) with $\left(\begin{array}{ll}a & b \\ c & d\end{array}\right)$ equal to minus the identity we see that $\phi$ is either even or odd, depending on whether $k$ is even or odd.

If all the coefficients $c_{n}(z)$ are contained in $K(\zeta)$, then we say that $\phi$ has a $K$-rational $q$-expansion. 
We denote by

$$
\wp(\tau, z)=\frac{1}{z^{2}}+\sum_{(m, n) \in \mathbf{Z}^{2} \backslash\{(0,0)\}}\left(\frac{1}{(z+m \tau+n)^{2}}-\frac{1}{(m \tau+n)^{2}}\right) \quad(\tau \in \mathcal{H}, z \in \mathbf{C})
$$

the Weierstrass $\wp$-function for the lattice $L_{\tau}=\mathbf{Z} \tau \oplus \mathbf{Z}$. This is a meromorphic Jacobi form of weight 2 and index zero; its $q$-expansion is given by

$\wp(\tau, z)=(2 \pi i)^{2}\left(\frac{1}{12}+\frac{\zeta}{(1-\zeta)^{2}}+\sum_{n \geq 1}\left(\sum_{d \mid n} d\left(\zeta^{d}+\zeta^{-d}-2\right)\right) q^{n}\right) \quad\left(|q|<|\zeta|<|q|^{-1}\right)$

(cf. e.g. 9, chap. 4, sect. 2]).

For an even integer $k \geq 4$ we denote by

$$
G_{k}(\tau)=\sum_{(m, n) \in \mathbf{Z}^{2} \backslash\{(0,0)\}} \frac{1}{(m \tau+n)^{k}} \quad(\tau \in \mathcal{H})
$$

the Eisenstein series of weight $k$ with respect to $\Gamma_{1}$. As usual, we let

$$
g_{2}(\tau):=60 G_{4}(\tau), g_{3}(\tau):=140 G_{6}(\tau)
$$

be the Weierstrass invariants attached to $\wp(\tau, \cdot)$.

We also write

$$
G_{2}(\tau)=\frac{\pi^{2}}{3}+\sum_{m \neq 0}\left(\sum_{n \in \mathbf{Z}} \frac{1}{(m \tau+n)^{2}}\right) \quad(\tau \in \mathcal{H})
$$

for the near-Eisenstein series of weight 2 with respect to $\Gamma_{1}$.

We sometimes also use the normalized functions

$$
Q:=12(2 \pi i)^{-4} g_{2}, R:=-216(2 \pi i)^{-6} g_{3}
$$

and

$$
P:=\frac{3}{\pi^{2}} G_{2}
$$

(Ramanujan's notation).

We let

$$
\Delta(\tau)=(2 \pi i)^{-12}\left(g_{2}^{3}(\tau)-27 g_{3}^{2}(\tau)\right)=q \prod_{n \geq 1}\left(1-q^{n}\right)^{24} \quad(\tau \in \mathcal{H})
$$

be the Ramanujan delta function which is a cusp form of weight 12 with respect to $\Gamma_{1}$.

We write

$$
j(\tau)=\frac{12^{3}}{(2 \pi i)^{12}} \frac{g_{2}^{3}(\tau)}{\Delta(\tau)}=\frac{1}{q}+744+196884 q+\ldots \quad(\tau \in \mathcal{H})
$$

for the usual modular invariant.

For each $n \in \mathbf{N}$ we define

$$
\wp_{n}(\tau, z):=\frac{1}{(2 n-1) !} \frac{\partial^{2 n-2}}{\partial z^{2 n-2}} \wp(\tau, z) .
$$

From the Laurent expansion of $\wp(\tau, \cdot)$ around $z=0$ it then easily follows that $\wp_{n}(\tau, \cdot)(n \geq 2)$ is the (uniquely determined) elliptic function with respect to $L_{\tau}$ which is holomorphic on $\mathbf{C} \backslash L_{\tau}$ and whose Laurent series around $z=0$ has the form

$$
\wp_{n}(\tau, z)=\frac{1}{z^{2 n}}+G_{2 n}(\tau)+\mathcal{O}\left(z^{2}\right) .
$$


The transformation formulas ii) for $\wp$ with $k=2$ and $m=0$ imply that $\wp_{n}$ is a meromorphic Jacobi form of weight $2 n$ and index zero. Clearly its $q$-expansion is Q-rational.

We normalize $\wp$ with respect to $\tau$ by setting

$$
w(\tau, z):=\left(-2^{7} \cdot 3^{5} \frac{g_{2}(\tau) g_{3}(\tau)}{(2 \pi i)^{12} \Delta(\tau)}\right) \wp(\tau, z) \quad(\tau \in \mathcal{H}, z \in \mathbf{C})
$$

so that $w$ is a meromorphic Jacobi form of weight zero and index zero with a Q-rational $q$-expansion.

The function $w(\tau, \cdot)$ classically is called the first Weber function attached to $L_{\tau}$, at least if $\tau \neq i, \rho$ (cf. 9, chap. 6, sect. 2]). (The factor $-2^{7} \cdot 3^{5}$ is traditionally inserted so that certain power series expansions have integral coefficients; we do not need this.)

According to [1, Satz 1] the field of meromorphic Jacobi forms of weight zero and index zero with a $K$-rational $q$-expansion is equal to $K(j, w)$.

Finally, following [5] we put

$$
\phi_{10,1}=\frac{1}{144}\left(R E_{4,1}-Q E_{6,1}\right)
$$

where $Q$ and $R$ are defined by (4) and $E_{4,1}$ resp. $E_{6,1}$ are the usual Jacobi-Eisenstein series of weight 4 resp. 6 and index 1 [5, chap. I, sects. 2 and 3]. Then $\phi_{10,1}$ is the unique Jacobi cusp form of weight 10 and index 1 whose Taylor expansion around $z=0$ has the form

$$
(2 \pi i)^{2} \Delta(\tau) z^{2}+\mathcal{O}\left(z^{4}\right)
$$

[5, chap. I, sect. 3]. The function $\phi_{10,1}(\tau, \cdot)$ vanishes doubly at $z=0$ and hence nowhere else on $\mathbf{C} \backslash L_{\tau}$ [5, chap. I, sect. 1, Thm. 1.2]. Also, $\phi_{10,1}$ has a Q-rational q-expansion [5, chap. I, sect. 3, formula (17)].

\section{Simple RAtionality RESUlts}

Proposition 1. Let $\phi$ be a meromorphic Jacobi form which has a K-rational qexpansion. Fix $\tau \in \mathcal{H}$ and suppose that $\phi(\tau, \cdot)$ is a non-zero meromorphic function on $\mathbf{C}$. Suppose that $z$ is a point in the divisor of $\phi(\tau, \cdot)$ and $z \notin L_{\tau}$. Then $\wp_{n}(\tau, z)$ is algebraic over $K\left(g_{2}(\tau), g_{3}(\tau)\right)$, for all $n \geq 1$, where $g_{2}$ and $g_{3}$ are defined by (2).

Proof. Replacing $\phi$ by $\frac{1}{\phi}$ it is sufficient to consider the case where $z$ is a zero of $\phi(\tau, \cdot)$.

It also suffices to consider the case $n=1$. Indeed, from the Laurent expansion of $\wp(\tau, z)$ at $z=0$ involving the Eisenstein series and from (7) it is easy to see by induction that $\wp_{n}$ is a monic polynomial in $\wp$ of degree $n$ and that the coefficient at $\wp^{\ell}(\ell<n)$ is in $\mathbf{Q}\left[g_{2}, g_{3}\right]$ (in fact, a modular form of weight $2 n-2 \ell$ ).

Let $k$ be the weight and $m$ be the index of $\phi$. Then

$$
\psi:=\frac{\phi^{24}}{\phi_{10,1}^{24 m} \Delta^{2 k-20 m}}
$$

with $\phi_{10,1}$ defined by (8) is a meromorphic Jacobi form of weight zero and index zero which outside of $L_{\tau}$ has the same zero divisor as $\phi$. Also $\psi$ has a $K$-rational $q$-expansion.

Therefore, by [1, Satz 1] (cf. section 2) one can write $\psi$ as a quotient of two polynomials in $w$ with coefficients in $K[j]$. Since $\{(\tau, z) \mid z \in \mathbf{C}\}$ by hypothesis is not contained in the zero or pole curve of $\phi$, it follows that $w(\tau, z)$ is algebraic over 
$K(j(\tau))$. Since $w(i, \cdot)=0$ and $w(\rho, \cdot)=0$, the latter hypothesis also guarantees that $\tau \neq i, \rho$, hence $g_{2}(\tau) g_{3}(\tau) \neq 0$, and our assertion follows.

Remark. For $n \in \mathbf{N}$ put

$$
w_{n}(\tau, z):=\left(-2^{7} \cdot 3^{5} \frac{g_{2}(\tau) g_{3}(\tau)}{(2 \pi i)^{12} \Delta(\tau)}\right)^{n} \wp_{n}(\tau, z) \quad(\tau \in \mathcal{H}, z \in \mathbf{C}) .
$$

Then $w_{n}$ is a meromorphic Jacobi form of weight zero and index zero, and the same argument as above shows that $w_{n}(\tau, z)$ is algebraic over $K(j(\tau))$.

The Theorem in the next section gives an identity between the values of $\wp_{n}(\tau, z)$ occurring in Proposition 1 and the Laurent coefficients of $\phi(\tau, \cdot)$ at $z=0$. It might therefore be worthwhile to note the following result which for the sake of simplicity we state only in the holomorphic case.

Proposition 2. Let $\phi$ be a holomorphic Jacobi form with a $K$-rational q-expansion. Denote by $\chi_{n}(\tau)(n \geq 0)$ its Taylor coefficients around $z=0$. Then $\chi_{n}$ is in $K\left[g_{2}, g_{3}, G_{2}\right]$ for all $n$, where $G_{2}$ is defined by (3).

Proof. Since $\phi$ is holomorphic, the coefficients of the $q$-expansion of $\phi$ are Laurent polynomials in $\zeta$ with coefficients in $K$. Therefore, taking derivatives with respect to $z$ and evaluating at $z=0$ shows that $(2 \pi i)^{-n} \chi_{n}$ is a Fourier series in $q$ with coefficients in $K$, and the same is true for $(2 \pi i)^{-n-\ell} \chi_{n}^{(\ell)}(n \geq 0, \ell \geq 0)$.

By [5, chap. I, sect. 3] there exists a sequence $\left(\xi_{n}\right)_{n \geq 0}$ of holomorphic modular forms of weight $k+n$ with respect to $\Gamma_{1}$ such that $\chi_{n}$ for every $n \geq 0$ can be expressed as a Q-linear combination of $(2 \pi i)^{\ell} \xi_{n-2 \ell}^{(\ell)}\left(0 \leq \ell \leq \frac{n}{2}\right)$, and conversely $\xi_{n}(n \geq 0)$ can be expressed as a $\mathbf{Q}$-linear combination of $(2 \pi i)^{\ell} \chi_{n-2 \ell}^{(\ell)}\left(0 \leq \ell \leq \frac{n}{2}\right)$. It follows that $(2 \pi i)^{-n} \xi_{n}$ has Fourier coefficients in $K$, hence $(2 \pi i)^{k} \xi_{n} \in K\left[g_{2}, g_{3}\right]$ for all $n$.

We now observe the well-known formulas

$$
\begin{aligned}
(2 \pi i)^{-1} Q^{\prime} & =\frac{1}{3}(P Q-R), \\
(2 \pi i)^{-1} R^{\prime} & =\frac{1}{2}\left(P R-Q^{2}\right)
\end{aligned}
$$

and

$$
(2 \pi i)^{-1} P^{\prime}=\frac{1}{12}\left(P^{2}-Q\right)
$$

(with $Q, R$ defined by (4) and $P$ by (5)). These formulas imply that the differential operators $(2 \pi i)^{\ell} \frac{d^{\ell}}{d \tau^{\ell}}$ leave $K\left[g_{2}, g_{3}, G_{2}\right]$ invariant. Our claim now follows.

\section{Average sums of special values}

The main result of the paper is the following

Theorem. Let $\phi$ be a meromorphic Jacobi form of index $m \in \mathbf{Z}$. Fix $\tau \in \mathcal{H}$ and suppose that $\phi(\tau, \cdot)$ is a non-zero meromorphic function on $\mathbf{C}$. Write

$$
\frac{z \frac{\partial}{\partial z} \phi(\tau, z)}{\phi(\tau, z)}=h_{\tau}-\sum_{n \geq 1} b_{n}(\tau) z^{n}
$$


with $h_{\tau}=\operatorname{ord}_{z=0} \phi(\tau, \cdot)$. Let $\wp_{n}(n \geq 1)$ be the functions defined by (6), with $\wp_{1}=\wp$. Then one has

$$
\begin{aligned}
b_{2 n}(\tau) & =\sum_{z \in \mathbf{C} / L_{\tau}, z \notin L_{\tau}} \operatorname{ord}_{z} \phi(\tau, \cdot) \wp_{n}(\tau, z) \\
& + \begin{cases}2 m G_{2}(\tau), & \text { if } n=1, \\
h_{\tau} G_{2 n}(\tau), & \text { if } n>1,\end{cases}
\end{aligned}
$$

where $G_{2}, G_{4}, \ldots$ are the Eisenstein series defined by (1) and (3).

Remarks. i) Note that clearly $b_{n}(\tau)$ is a polynomial with integral coefficients in

$$
\frac{\chi_{1}(\tau)}{\chi_{0}(\tau)}, \ldots, \frac{\chi_{n}(\tau)}{\chi_{0}(\tau)}
$$

where

$$
\phi(\tau, z)=\sum_{n \geq 0} \chi_{n}(\tau) z^{n+h_{\tau}}
$$

is the Laurent expansion of $\phi(\tau, \cdot)$ at $z=0$. In fact, according to [3, proof of Theorem 3] one has the explicit formula

$$
b_{n}(\tau)=F_{n}\left(\frac{\chi_{1}(\tau)}{\chi_{0}(\tau)}, \ldots, \frac{\chi_{n}(\tau)}{\chi_{0}(\tau)}\right)
$$

where

$$
\begin{gathered}
F_{n}\left(X_{1}, \ldots, X_{n}\right):=n \sum_{\substack{m_{1}, \ldots, m_{n} \geq 0 \\
m_{1}+2 m_{2}+\cdots+n m_{n}=n}}(-1)^{m_{1}+\cdots+m_{n}} \frac{\left(m_{1}+\cdots+m_{n}-1\right) !}{m_{1} ! \ldots m_{n} !} \\
\cdot X_{1}^{m_{1}} \ldots X_{n}^{m_{n}} .
\end{gathered}
$$

ii) Notice that evaluating the integral (10) below in the same way with $\wp_{n}$ replaced by a partial derivative of $\wp$ with respect to $z$ of odd order would lead only to an obvious zero identity, since for $\phi$ a Jacobi form the function $\frac{\frac{\partial}{\partial z} \phi(\tau, z)}{\phi(\tau, z)}$ is always odd with respect to $z$ and the corresponding $\wp_{n}$ also would be odd.

iii) In the proof of the Theorem, only the relevant transformation formula of $\phi$ under $\mathbf{Z}^{2}$ and not under $\Gamma_{1}$ will enter. Hence a corresponding statement would, e.g., also be true with $\phi$ replaced by $L_{m} \phi$, where $L_{m}:=8 \pi i m \frac{\partial}{\partial \tau}-\frac{\partial^{2}}{\partial z^{2}}$ is the heat operator (which in general does not map Jacobi forms to themselves). Note that $L$ sends even (odd) functions to even (odd) functions with respect to $z$.

Proof of the Theorem. The argument is similar to the proof of Theorem $1.2 \mathrm{on}$ p. 10 of [5]. Let $\mathcal{F}_{\tau}$ be a closed fundamental parallelogram for the action of $L_{\tau}$ on $\mathbf{C}$ whose boundary does not contain any zeros or poles of $\phi(\tau, \cdot)$ nor any points of $L_{\tau}$. For convenience, let us also suppose that $0 \in \mathcal{F}_{\tau}$. We consider the integral

$$
\frac{1}{2 \pi i} \int_{\partial \mathcal{F}_{\tau}} \frac{\frac{\partial}{\partial z} \phi(\tau, z)}{\phi(\tau, z)} \wp_{n}(\tau, z) d z .
$$

Since

$$
\frac{1}{2 \pi i} \frac{\frac{\partial}{\partial z} \phi(\tau, z)}{\phi(\tau, z)}
$$


is invariant under $z \mapsto z+1$ and changes by $2 m$ when one replaces $z$ by $z+\tau$, and $\wp_{n}$ is invariant under these substitutions, the value of (10) is equal to

$$
2 m \int_{z_{0}}^{z_{0}+1} \wp_{n}(\tau, z) d z
$$

where $z_{0}$ is an appropriate point in $\mathbf{C} \backslash L_{\tau}$ and the integration is along the line between $z_{0}$ and $z_{0}+1$ with positive orientation.

Suppose that $n>1$. Then by the definition of $\wp_{n}$ and since $\wp(\tau, \cdot)$ has period 1 , we obtain for (11) the value zero.

On the other hand, if $n=1$, we get for (11) the value $-2 m \eta_{\tau}(1)$. Here

$$
\eta_{\tau}: L_{\tau} \rightarrow \mathbf{C}, \omega \mapsto \zeta(\tau, z+\omega)-\zeta(\tau, z) \quad\left(\text { any } z \in \mathbf{C} \backslash L_{\tau}\right)
$$

is the Weierstrass eta function for $L_{\tau}$, and $\zeta(\tau, z)(\tau \in \mathcal{H}, z \in \mathbf{C})$ is the usual Weierstrass zeta function for $L_{\tau}$ so that

$$
\frac{\partial}{\partial z} \zeta(\tau, z)=-\wp(\tau, z)
$$

We can also evaluate (10) by the residue theorem, taking into account that $\wp_{n}(\tau, \cdot)$ is holomorphic outside of $L_{\tau}$ and that for $n \geq 2$ its Laurent series at $z=0$ is given by (7) (if $n=1$, of course, we have $\wp(\tau, \cdot)=\frac{1}{z^{2}}+\mathcal{O}\left(z^{2}\right)$ ). We then obtain for (10) the value

$$
\sum_{z \in \mathbf{C} / L_{\tau}, z \notin L_{\tau}} \operatorname{ord}_{z} \phi(\tau, \cdot) \wp_{n}(\tau, z)+\left(\delta_{n} h_{\tau} G_{2 n}(\tau)-b_{2 n}(\tau)\right)
$$

where $\delta_{n}$ is 1 or zero according as $n>1$ or $n=1$.

We therefore find the identity

$$
\begin{aligned}
b_{2 n}(\tau)= & \sum_{z \in \mathbf{C} / L_{\tau}, z \notin L_{\tau}} \operatorname{ord}_{z} \phi(\tau, \cdot) \wp_{n}(\tau, z) \\
& + \begin{cases}2 m \eta_{\tau}(1), & \text { if } n=1, \\
h_{\tau} G_{2 n}(\tau), & \text { if } n>1 .\end{cases}
\end{aligned}
$$

This proves the assertion of the Theorem for $n>1$. To prove it for $n=1$, it suffices to show that

$$
\eta_{\tau}(1)=G_{2}(\tau) .
$$

Identity (13) for example is given in [8 pp. 166-167]. It is also an easy consequence of (12) in the special case of the Jacobi cusp form $\phi_{10,1}$ of weight 10 and index 1 defined by (8). Indeed, recall that $\phi_{10,1}(\tau, \cdot)$ vanishes doubly at $z=0$ and nowhere else on $\mathbf{C} \backslash L_{\tau}$, and that its Taylor expansion around $z=0$ is given by

$$
\phi_{10,1}(\tau, z)=-(2 \pi)^{2} \Delta(\tau) z^{2}+\frac{4}{3} \pi^{4} P(\tau) \Delta(\tau) z^{4}+\mathcal{O}\left(z^{6}\right),
$$

where $P$ is defined by (5) (the latter formula follows from the Taylor expansions of $E_{4,1}$ and $E_{6,1}$ given in [5, chap. III, sect. 8, p. 94]). Since

$$
F_{2}\left(X_{1}, X_{2}\right)=X_{1}^{2}-2 X_{2}
$$

this implies (13).

This finishes the proof of the Theorem. 


\section{Product expansions}

We shall make some short remarks about product expansions of Jacobi forms in terms of the functions $1-z^{n}(n \geq 1)$.

Let

$$
f(z)=\sum_{n \geq 0} a_{n} z^{n+h}
$$

be a power series holomorphic in a small punctured neighborhood of $z=0$, with $h \in \mathbf{Z}$ and $a_{0} \neq 0$. Then as is easy to see [4, sect. 2] (cf. also [3, Propos. 2.1]), $f$ has a product expansion

$$
f(z)=a_{0} z^{h} \prod_{n \geq 1}\left(1-z^{n}\right)^{c_{n}}
$$

convergent in a small $\epsilon$-neighborhood of $z=0$ (contained in the unit disc). Here we use the convention that complex powers are defined by the principal branch of the complex logarithm. Moreover, for $|z|<\epsilon$ one has the identity

$$
\frac{z f^{\prime}(z)}{f(z)}=h-\sum_{n \geq 1}\left(\sum_{d \mid n} d c_{d}\right) z^{n}
$$

Therefore, bearing in mind Remark i) after the Theorem, the left-hand side of (9) could also be rewritten in terms of the exponents $c_{n}(\tau)$ of the $z$-product expansion of $\phi(\tau, \cdot)$. Note that when $\tau$ ranges over $\mathcal{H}, \phi(\tau, \cdot)$ in general will have zeros arbitrarily close to $z=0$, and so the convergence in $z$ of this product for each fixed $\tau$ will depend on the chosen $\tau$.

As an example, let us again consider the case $\phi=\phi_{10,1}$. For $\tau \in \mathcal{H}$ let

$$
\delta_{\tau}:=\min \left\{|m \tau+n| \mid(m, n) \in \mathbf{Z}^{2} \backslash\{(0,0)\}\right\} .
$$

Then from the Theorem one easily finds

Proposition 3. For the function $\phi_{10,1}$ defined by (8), one has

$$
\phi_{10,1}(\tau, z)=-(2 \pi)^{2} \Delta(\tau) z^{2} \prod_{n \geq 1}\left(1-z^{2 n}\right)^{c_{n}(\tau)} \quad\left(\tau \in \mathcal{H},|z|<\min \left\{\delta_{\tau}, 1\right\}\right)
$$

where

$$
c_{n}(\tau):=\frac{1}{n} \sum_{d \mid n} \mu\left(\frac{n}{d}\right) G_{2 d}(\tau),
$$

$\mu$ is the Möbius function and the Eisenstein series $G_{2}, G_{4}, \ldots$ are defined by (1) and (3).

\section{ACKNOWLEDGEMENTS}

During the preparation of this work, the first author was partially supported by COM2MAC and would like to thank KIAS for its hospitality. The second author would like to thank KIAS for its financial support and hospitality. 


\section{REFERENCES}

1. R. Berndt, Zur Arithmetik der elliptischen Funktionenkörper höherer Stufe, J. Reine Angew. Math. 326 (1981), 79-94 MR 84h:10029a

2. R.E. Borcherds, Automorphic forms on $O_{s+2,2}(\mathbf{R})$ and infinite products, Invent. Math. 120 (1995), 161-213 MR 96j:11067

3. J.H. Bruinier, W. Kohnen and K. Ono, The arithmetic of the values of modular functions and the divisors of modular forms, to appear in Compos. Math.

4. W. Eholzer and N.-P. Skoruppa, Product expansions of conformal characters, Phys. Lett. B 388 (1996), 82-89 MR 97k:81132

5. M. Eichler and D. Zagier, The theory of Jacobi forms, Progress in Math. vol. 55, Birkhäuser: Boston 1985 MR 86j:11043

6. B. Gross and D. Zagier, Heegner points and derivatives of $L$-series, Invent. Math. 84 (1986), 225-320 MR 87j:11057

7. B. Gross and D. Zagier, On singular moduli, J. reine Angew. Math. 355 (1985), 191-220 MR 86j:11041

8. N. Katz, p-adic properties of modular schemes and modular forms. In: Modular Functions of One Variable III (eds.: W. Kuyk and J.-P. Serre), pp. 69-122, LNM 350, Springer: Berlin Heidelberg New York, 1973 MR 56:5434

9. S. Lang, Elliptic functions, Addison-Wesley: London 1973 MR 53:13117

10. D. Zagier, Traces of singular moduli, preprint 2000

Department of Mathematics, Pohang Institute of Science and Technology, Pohang 790-784, KOREA

E-mail address: yjc@postech.ac.kr

Mathematisches Institut, Universität Heidelberg, INF 288, D-69120 Heidelberg, GerMANY

E-mail address: winfried@mathi.uni-heidelberg.de 